\title{
ON THE INFINITESIMAL GENERATOR OF A SEMIGROUP OF POSITIVE TRANSFORMATIONS WITH LOCAL CHARACTER CONDITION ${ }^{1}$
}

\author{
SOLOMON LEADER
}

The study of one-dimensional diffusion processes leads to the consideration of the following structure.

Let $\left\{T_{t}\right\}(t>0)$ be a one-parameter semigroup [2] of linear operators on the space $C$ of bounded continuous functions on a closed interval $I$ satisfying the conditions:

(a) If $f(x) \geqq 0$ for all $x$ in $I$, then $T_{t} f(x) \geqq 0$ for all $x$ and all $t$ (positivity).

(b) If $f$ vanishes throughout a neighborhood of $x$, then $T_{t} f(x)$ $=o(t)$ (local character).

(c) $T_{t} 1=1$ for all $t$ (conservation of mass).

Let $\Omega$ be the infinitesimal generator of $\left\{T_{t}\right\}$. That is,

$$
\Omega f(x)=\lim _{t \rightarrow 0+} \frac{T_{t} f(x)-f(x)}{t}
$$

defined on the set $D$ of those $f$ in $C$ for which the limit exists uniformly in $x$. We shall also consider the expression (1) wherever the limit exists pointwise.

In this paper the form of $\Omega$ at a point $x$ is determined, but the problem of characterizing $\Omega$ in the large is left open.

W. Feller [1] has shown that if the functions in $D$ are twice differentiable, then, with the exception of certain singular points, $\Omega$ corresponds to a differential operator of the form

$$
\Omega f(x)=a(x) \frac{d^{2} f(x)}{d x^{2}}+b(x) \frac{d f(x)}{d x}
$$

where $a(x) \geqq 0$. However, Feller was dissatisfied with the arbitrary injection of differentiability conditions since the conditions (a), (b), and (c) are independent of the metric on $I$. He conjectured that, with only the conditions (a), (b), and (c), $\Omega$ must be essentially a second order differential operator.

Consider a fixed point $x$ in $I$. We shall consider only those functions $f$ for which $f(x)=0$. This is no real restriction since (c) implies

Received by the editors May 6, 1953 and, in revised form, October 22, 1953.

${ }^{1}$ Research sponsored by the Office of Ordnance Research. The author is grateful to Professor William Feller of Princeton University for his assistance and advice. 
$\Omega f=\Omega(f-\lambda)$ wherever $\Omega f$ exists. The expression "near $x$ " will be used to mean "in some neighborhood of $x$." The notation $f=o(h)$ will be used whenever, for arbitrary $\epsilon>0,|f(z)| \leqq \epsilon|h(z)|$ for all $z$ near $x$. The functions $f_{-}$and $f_{+}$, defined by

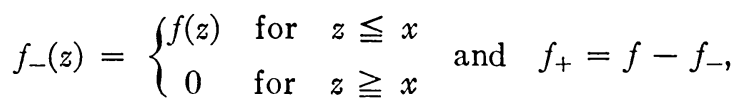

will also be useful.

We begin with two fundamental lemmas due to Feller [1].

Lemma A. If $f \geqq 0$ near $x$ and $\Omega f(x)$ exists, then $\Omega f(x) \geqq 0$. Thus, $\Omega f(z) \geqq 0$ if $f$ has a local minimum at $z$ and $\Omega f(z) \leqq 0$ if $f$ has a local maximum at $z$.

Proof. $|f|-f=0$ near $x$. Hence $T_{t}|f|(x)-T_{t} f(x)=o(t)$ by (b). Since $T_{t}|f| \geqq 0$ by (a), and $\Omega f(x)=\lim _{t \rightarrow 0} T_{t} f(x) / t=\lim _{t \rightarrow 0} T_{t}|f|(x) / t$, $\Omega f(x) \geqq 0$.

Lemma B. If $\Omega v(x)$ exists, $v \geqq 0$ near $x$, and $f=o(v)$, then $\Omega f(x)$ exists and $\Omega f(x)=0$.

Proof. For arbitrary $\epsilon>0,-\epsilon v \leqq f \leqq \epsilon v$ near $x$. From (b), $-\epsilon T_{t} v(x)$ $\leqq T_{t} f(x)+o(t) \leqq \epsilon T_{t} v(x)$. Dividing by $t$ and letting $t$ approach 0 ,

$$
-\epsilon \Omega v(x) \leqq \liminf _{t \rightarrow 0} \frac{T_{t} f(x)}{t} \leqq \limsup _{t \rightarrow 0} \frac{T_{t} f(x)}{t} \leqq \epsilon \Omega v(x) .
$$

Since $\epsilon$ may be arbitrarily small, the conclusion holds.

In order to introduce a metric we prove

THEOREM 1. If $f$ is in $D$ and $\Omega f(x) \neq 0$, then $f$ is strictly monotone in a right (left) neighborhood of $x$.

Proof. Suppose $f$ is not strictly monotone in any right neighborhood of $x$. Then, by induction, we can choose a double sequence of points $\left\{y_{n}, z_{n}\right\}$ such that $y_{n} \downarrow x, z_{n} \downarrow x, f\left(y_{n}\right)=f\left(z_{n}\right)$, and $x \leqq y_{n+1}$ $<z_{n+1}<y_{n}<z_{n}$ for all $n$. Thus, there exist points $r_{n}$ and $s_{n}$ in the interior of $\left(y_{n+1}, z_{n}\right)$ such that $f$ has a local minimum at $r_{n}$ and a local maximum at $s_{n}$. By Lemma $A$, we have $\Omega f\left(r_{n}\right) \geqq 0$ and $\Omega f\left(s_{n}\right) \leqq 0$. Since $r_{n} \downarrow x, s_{n} \downarrow x$, and since $\Omega f$ is continuous for $f$ in $D$, it follows that $\Omega f(x)=0$, a contradiction. A symmetric argument gives the theorem for a left neighborhood.

An immediate result of Theorem 1 is

Corollary 1. If $f$ is in $D$ and the zeros of $f$ are dense at $x$, then $\Omega f(x)=0$. 
If there is a function $g$ in $D$ with $\Omega g(x) \neq 0$, then every $f$ in $D$ has right and left derivatives (not necessarily finite) at $x$ with respect to $g$. The existence of unilateral derivatives is given by

Theorem 2. If $f$ and $g$ are in $D$ and $\Omega g(x) \neq 0$, then

$$
\liminf _{z \rightarrow x+} \frac{f(z)}{g(z)}=\limsup _{z \rightarrow x+} \frac{f(z)}{g(z)}
$$

and

$$
\liminf _{z \rightarrow x-} \frac{f(z)}{g(z)}=\limsup _{z \rightarrow x-} \frac{f(z)}{g(z)} .
$$

Proof. Let $P$ denote the set of real numbers $\rho$ for which $\rho g_{+} \leqq f_{+}$ near $x$, and $Q$ denote the set for which $f_{+} \leqq \rho g_{+}$near $x$. Then $\rho^{-}$ $=\sup _{P} \rho$ and $\rho^{+}=\inf _{Q} \rho$ are the lower and upper right derivatives of $f$ with respect to $g$. Suppose $\rho^{-} \neq \rho^{+}$. Then consider any finite $\rho$ such that $\rho^{-}<\rho<\rho^{+}$and $\rho \neq \Omega f(x) / \Omega g(x)$. Such a $\rho$ belongs to neither $P$ nor $Q$. Thus $f_{+}-\rho g_{+}(z)<0$ at a set of points dense at $x$ and $f_{+}-\rho g_{+}(z)$ $>0$ at a dense set. Hence, the zeros of $f-\rho g$ are dense at $x$. By Corollary $1, \Omega f(x)=\rho \Omega g(x)$, a contradiction. Therefore, $\rho^{-}=\rho^{+}$. A similar proof holds for the left derivatives.

We now seek to express $\Omega$ in terms of such unilateral derivatives.

Theorem 3. Let $f$ and $g$ belong to $D$. If either $f_{+}=o(g)$ and $f_{+} \geqq 0$ near $x$ or $f_{-}=o(g)$ and $f_{-} \geqq 0$ near $x$, then $\Omega f(x) \geqq 0$.

Proof. If $g$ has its zeros dense at $x$ from the right, then $f$ does likewise for $f_{+}=o(g)$, so $\Omega f(x)=0$ by Corollary 1. Otherwise we may assume that $g>0$ in a deleted right neighborhood of $x$. Then for each $\epsilon>0,0 \leqq f_{+} \leqq \epsilon g_{+}$near $x$. For $z$ in an arbitrary deleted right neighborhood of $x$ we may set $\epsilon=f(z) / g(z)$. Then $f_{+}-\epsilon g_{+} \leqq 0$ near $x$ and $f-\epsilon g(x)=f-\epsilon g(z)=0$, so $f-\epsilon g$ has a local minimum at some point $y$, $x<y<z$. By Lemma A, $\Omega(f-\epsilon g)(y) \geqq 0$, so $\Omega f(y) \geqq \epsilon \Omega g(y)$. As $z$ converges to $x, \epsilon$ converges to 0 and $y$ converges to $x$ giving $\Omega f(x) \geqq 0$. A similar proof gives the theorem with $f_{+}$replaced by $f_{-}$.

We can now extend Lemma $B$ to give

Theorem 4. Suppose either $g \geqq 0$ or $f g \geqq 0$ near $x$ for $f$ and $g$ in $D$. Then $f=o(g)$ implies $\Omega f(x)=0$.

Proof. Suppose $\Omega f(x) \neq 0$. Then we cannot have $g \geqq 0$ near $x$, for this would contradict Lemma B. Hence, $f g \geqq 0$ near $x$ with $g$ having neither a maximum nor minimum at $x$. By Theorem $1, f$ is strictly monotone near $x$. Thus, either $f_{-} \leqq 0$ and $f_{+} \geqq 0$ or $f_{-} \geqq 0$ and $f_{+} \leqq 0$ 
near $x$. By Theorem $3, \Omega f(x)=0$, contradicting our initial assumption.

By means of the preceding theorem, Theorem 2 can be sharpened to give

Theorem 5. If $u$ is in $D, \Omega u(x) \neq 0$, and $u$ is monotone near $x$, then every $f$ in $D$ has finite unilateral derivatives with respect to $u$.

Proof. Suppose $f$ has an infinite right derivative, $\sigma_{+}= \pm \infty$. Then $f$ must have a finite left derivative, $\sigma_{-}$. Otherwise, $u=o(f)$ and Theorem 4 would give $\Omega u(x)=0$, a contradiction.

Choose $\sigma$ so that $\sigma_{-}<\sigma<\infty$ if $\sigma_{+}=\infty$ and $-\infty<\sigma<\sigma_{-}$if $\sigma_{+}=-\infty$. Let $v=f-\sigma u$. Then, since $u_{+}=o(v)$, Lemma B gives $\Omega u_{+}(x)=0$. So $\Omega u(x)=\Omega u_{-}(x)$. But $\Omega u_{-}(x) \leqq 0$ if $u$ is increasing, by Lemma A. So $\Omega u(x)<0$. However, $u_{+} \geqq 0$ near $x$ and $u_{+}=o(f)$ implying $\Omega u(x)>0$ by Theorem 3 , a contradiction.

A similar proof holds for the left derivative.

We can now express $\Omega$ at $x$ in terms of an increasing function $u$ and a function $v$ having a minimum at $x$.

Theorem 6. At $x$ one of the following four cases must hold:

(I) $\Omega f(x)=0$ for all $f$ in $D$.

(II) For all $u$ in $D$ monotone near $x, \Omega u(x)=0$. There exists $v$ in $D$ with $v \geqq 0$ near $x$ and $\Omega v(x)>0$. Then, for each $f$ in $D$ there exists a number $\rho$ such that

$$
f=\rho v+o(v)
$$

and

$$
\Omega f(x)=\rho \Omega v(x) .
$$

(III) For all $v$ in $D$ with $v \geqq 0$ near $x, \Omega v(x)=0$. There exists $u$ in $D$ with $u$ strictly increasing near $x$ and $\Omega u(x) \neq 0$. Then, for each $f$ in $D$ there exists a number $\sigma$ such that

$$
f=\sigma u+o(u)
$$

and

$$
f(x)=\sigma \Omega u(x) .
$$

(IV) There exist $u$ and $v$ in $D$ with $u$ strictly increasing near $x$, $\Omega u(x) \neq 0, v \geqq 0$ near $x$, and $\Omega v(x)>0$ such that each $f$ in $D$ can be expressed in the form

$$
f=\sigma u+\rho v+h
$$

where $h=o(u)=o(v)$. Then 


$$
\Omega f(x)=\sigma \Omega u(x)+\rho \Omega v(x) .
$$

Proof. If case (I) does not hold, there exist functions $g$ in $D$ with $\Omega g(x) \neq 0$. Such functions are either strictly monotone near $x$ or have a maximum (or minimum) at $x$, by Theorem 1 . If all such $g$ are of the latter type, we have case (II); if the former type, we have case (III).

In case (II) consider any $f$ in $D$. If $f$ had no derivative with respect

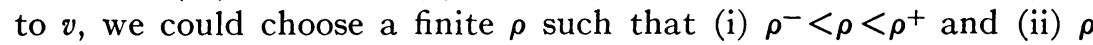
$\neq \Omega f(x) / \Omega v(x)$. By (ii), $\Omega(f-\rho v)(x) \neq 0$. So $f-\rho v$ is unilaterally monotone near $x$, by Theorem 1. By Theorem 2, $f-\rho v$ has unilateral derivatives with respect to $v$ and these derivatives, $\rho^{-}-\rho$ and $\rho^{+}-\rho$, are of opposite sign, by (i). Thus, since $v \geqq 0$ near $x, f-\rho v$ is monotone near $x$. From the assumptions of case (II), $\Omega(f-\rho v)(x)=0$ which contradicts (ii). Hence, (2) holds. (3) follows from Lemma B.

In case (III) each $f$ has a finite derivative $\sigma$ with respect to $u$. Otherwise, there would exist a finite $\sigma$ such that (i) $\sigma^{-}<\sigma<\sigma^{+}$and (ii) $\sigma \neq \Omega f(x) / \Omega u(x)$. Since $f$ has finite unilateral derivatives $\sigma_{-}, \sigma_{+}$ with respect to $u$ by Theorem 5, (i) implies either $f-\sigma u \geqq 0$ or $f-\sigma u$ $\leqq 0$ near $x$. Hence, (III) gives $\Omega(f-\sigma u)(x)=0$ which contradicts (ii). Thus, we obtain (4) with $\sigma=\sigma_{-}=\sigma_{+}$. If $f-\sigma u$ is not monotone near $x$, then $\Omega(f-\sigma u)(x)=0$ by (III). If $f-\sigma u$ is monotone near $x$, then $\Omega(f-\sigma u)(x)=0$ by Theorem 4 . Hence, (5) holds.

If the unilaterally monotone functions $g$ for which $\Omega g(x) \neq 0$ are of two types, some monotone near $x$ and others with extreme values at $x$, then we may choose a function of each type. In particular, choose $u$ such that $u$ is increasing near $x$ and $\Omega u(x) \neq 0$, and choose $v$ such that $v \geqq 0$ near $x$ and $\Omega v(x)>0$. We then have (IV), which we treat in two cases: one in which $v$ has a derivative with respect to $u$ and the other in which $v$ has no derivative with respect to $u$.

Case (IV-1). $v=o(u)$. In this case every $f$ in $D$ has a derivative $\sigma$ with respect to $u$. For, suppose $\sigma_{-} \neq \sigma_{+}$. Then $s=f-2^{-1}\left(\sigma_{-}+\sigma_{+}\right) u$ has unilateral derivatives $2^{-1}\left(\sigma_{-}-\sigma_{+}\right)$and $2^{-1}\left(\sigma_{+}-\sigma_{-}\right)$which differ in sign. So $s$ has a maximum (or minimum) at $x$ and $v=o(s)$. Lemma B gives $\Omega v(x)=0$, a contradiction.

Let $s=f-\sigma u$, so $s=o(u)$. If $s$ had unequal derivatives $\left(\rho^{-}<\rho^{+}\right)$ with respect to $v$, we could choose $\rho$ such that $\rho^{-}<\rho<\rho^{+}$and $\rho$ $\neq \Omega s(x) / \Omega v(x)$. Thus, $\Omega(s-\rho v)(x) \neq 0$. So $s-\rho v$ is unilaterally monotone near $x$, by Theorem 1 . By Theorem $2, s-\rho v$ has unilateral derivatives with respect to $v$ and these derivatives, $\rho^{-}-\rho$ and $\rho^{+}-\rho$, are of opposite sign. Since $v \geqq 0$ near $x, s-\rho v$ is monotone near $x$. But $s-\rho v=o(u)$, so $\Omega(s-\rho v)(x)=0$, by Theorem 4 , a contradiction. Hence, let $\rho=\rho^{-}=\rho^{+}$which must be finite. For, if $\rho=\infty($ or $\rho=-\infty)$, then 
$s \geqq 0$ (or $s \leqq 0$ ) near $x$, since $v \geqq 0$ near $x$, and $v=o(s)$. Theorem 4 would give $\Omega v(x)=0$, a contradiction. We thus have (6) for case (IV-1).

Case (IV-2). $v$ has no derivative with respect to $u\left(\beta_{-}<\beta_{+}\right)$. We may assume $\beta_{+}=1$ and $\beta_{-}=-1$. For, given any $w$ in $D$ with $w \geqq 0$ near $x, \Omega w(x)>0$, and unilateral derivatives $\left(\alpha_{-}<\alpha_{+}\right)$with respect to $u$, we can define

$$
v=\frac{2}{\alpha_{+}-\alpha_{-}}\left\{w-\frac{1}{2}\left(\alpha_{+}+\alpha_{-}\right) u\right\} .
$$

Thus, $v$ has $\beta_{+}=1, \beta_{-}=-1$ and $v \geqq 0$ near $x$. Choose $\beta$ such that -1 $<\beta<1$ with sign such that $\beta \Omega u(x)>0$. Then, $v-\beta u \geqq 0$ near $x$, so $\Omega(v-\beta u)(x) \geqq 0$ by Lemma A. Thus $\Omega v(x)>0$.

Now, for $f$ in $D$ with unilateral derivatives $\sigma_{-}, \sigma_{+}$with respect to $u$, let $\sigma=2^{-1}\left(\sigma_{+}+\sigma_{-}\right)$and $\rho=2^{-1}\left(\sigma_{+}-\sigma_{-}\right)$. Then it is easily verified by taking right and left derivatives that $f-\sigma u-\rho v=o(u)=o(v)$, thus giving (6). (7) follows from Lemma B.

Thus, $\Omega$ will correspond to a second order differential operator at $x$ whenever it is possible to choose $u$ and $v$ such that $u^{2}=v+o(v)$.

\section{REFERENCES}

1. W. Feller, On positivity preserving semigroups of transformations on $C\left[r_{1}, r_{2}\right]$, Annales de la Société Polonaise de Mathématique (1952).

2. E. Hille, Functional analysis and semi-groups, Amer. Math. Soc. Colloquium Publications, vol. 31, New York, 1948.

RUTGERS UNIVERSITY 